\title{
Vestibular influence on water maze retention: transient whole body rotations improve the accuracy of the cue-based retention strategy
}

\author{
Anne-Marie T. McGauran ${ }^{\mathrm{a}}$, Shane M. O'Mara ${ }^{\mathrm{b}}$, Sean Commins ${ }^{\mathrm{a}, *}$ \\ ${ }^{\text {a }}$ Department of Psychology, National University of Ireland, Maynooth, Co. Kildare, Ireland \\ ${ }^{\mathrm{b}}$ Department of Psychology and Trinity College Institute of Neuroscience, University of Dublin, Trinity College, Dublin 2, Ireland
}

Received 28 June 2004; received in revised form 30 July 2004; accepted 6 August 2004

Available online 19 September 2004

\begin{abstract}
Spatial learning in the water maze is thought to rely both on distal cues and vestibular information [Aggleton JP, Vann SD, Oswald CJP, Good M. Identifying cortical inputs to the rat hippocampus that subserves allocentric spatial processes: a simple problem with a complex answer. Hippocampus 2000;10:466-74; Pearce JM. Animal learning and cognition: an introduction. UK: Psychology Press; 1997]. Experiment 1 demonstrates that while water maze retention relies primarily on cue-platform based associations, this strategy is not precise, as animals tend to focus at the side of the pool. In experiment 2, we demonstrate that vestibular rotation eliminates this inaccuracy. These experiments highlight the importance of both cue and vestibular information for accurate retention of the water maze.
\end{abstract}

(C) 2004 Elsevier B.V. All rights reserved.

Keywords: Retention; Vestibular rotation; Spatial memory; Water maze; Distal cues

Research in spatial navigation has investigated the use of many different strategies in the formation of spatial memories for an environment $[6,11]$. The influence of both internally generated information and external cues in navigation have been well documented, with researchers arguing the importance of one or both in successful navigation [1,13]. Animals may use information obtained from internal, vestibular cues when locating their position within an environment, focusing on self-motion cues (path integration) or their position in relation to their starting point (dead reckoning) ([7], see also [10]). On the other hand, much research has highlighted the importance of external, allocentric information in spatial navigation $[11,19]$. Morris, for example, determined that navigating rats will follow distal visual cues to locate a hidden platform [11]. When these cues are rotated, animals will rotate their search for the platform concurrently in relation to these cues. While most research into spatial navigation has examined acquisition of the water maze, the study of retention

\footnotetext{
* Corresponding author. Tel.: +353 1 7086182; fax: +35317084767

E-mail address: sean.commins@may.ie (S. Commins).
}

has been relatively ignored, despite its importance in understanding the formation of long-term spatial memories [5]. We have recently demonstrated that, similar to acquisition, animals during long-term retention prefer to follow allocentric, rather than egocentric strategies [9]. Rotation of distal cues by $180^{\circ}, 7$ days post-acquisition, for example, leads animals to rotate their searching strategy during the retention trial. In experiment 1 , we attempt to extend these finding by providing an in-depth analysis of the cue-based strategy used during retention.

Male Wistar rats (200-300 g; Bioresources Unit, University of Dublin, Trinity College), aged approximately 3 months were used for the experiments in this study. All rats were housed two or three subjects per cage and were given free access to food and drink. Animals were kept in a temperaturecontrolled room, which was maintained on a fixed light-dark cycle (07:00-19:00 h). Testing was carried out during the light phase, and all rats were well handled before experimentation.

A standard water maze procedure was used. This was a black circular pool $(1.7 \mathrm{~m}$ diameter; $35 \mathrm{~cm}$ deep) filled to 
$31 \mathrm{~cm}$. Rats could escape the water by climbing onto a platform $(29 \mathrm{~cm} \times 9 \mathrm{~cm})$ located in the north eastern quadrant of the pool, and submerged $2 \mathrm{~cm}$ below water surface so that it was invisible at water level. A curtain surrounded the entire maze at a distance of $50 \mathrm{~cm}$ from the pool wall. Distal visual cues included two $(60 \mathrm{~W})$ lights suspended from the ceiling (on the inside of the curtain) at a distance of $75 \mathrm{~cm}$ from the pool wall and at angles of approximately $60^{\circ}$. A rectangular sheet of black paper $(55 \mathrm{~cm} \times 81 \mathrm{~cm})$ attached to the curtain on the eastern side of the pool was also used as a distal cue. One light bulb was suspended from the NW corner, and the other from the NE position. Escape latencies were recorded during each trial using a computerised digital tracking system (EthoVision: Noldus Information Technology, Wageningen, The Netherlands).

Rats were trained for four trials per day for 4 days: they were placed into the maze from a pseudo-random starting point (N, S, E, W). If they did not locate the platform after $60 \mathrm{~s}$ had elapsed, they were led to the platform by the researcher. The animal could then remain on the platform for $20 \mathrm{~s}$. The inter-trial interval was $10 \mathrm{~s}$ which was spent by the animals in a small open-top box outside the testing arena.

Retention was assessed 7 days post-acquisition. Rats were randomly assigned to one of two groups: controls $(n=15)$ or cue-rotated group $(n=15)$. Prior to the retention trial all animals were carefully carried to the testing arena in an opentop box, within which they were free to walk and observe their surroundings. Furthermore to ensure all animals were not disoriented before the retention trial all animals were carried along the same trajectory from their home cage to the testing arena as they had been during the acquisition period. Both groups were placed into the water maze from a fixed starting position at the NW quadrant. Retention for both groups was assessed by removing the platform and allowing the rats to swim for $60 \mathrm{~s}$ around the maze so that their swimming pattern could be observed. For the control group (CT), the distal-cue layout remained the same as during the acquisition phase. For the cue-rotated group (CR), the distal cues were rotated by $180^{\circ}$, so that the sheet of black paper was now positioned on the western side of the maze, one light was suspended from the SW corner, and the other from the SE position.

Measures of retention included the analysis of percentage time spent in each of the four quadrants (NE, NW, SE, SW) during the initial $30 \mathrm{~s}$ of the retention phase. The first $30 \mathrm{~s}$ were chosen, as we have previously shown that retention is sensitive to disruption after this time period [9]. Percentage time spent in the platform area was calculated (this area was defined by a circular area centred at the platform with a radius of approximately $27 \mathrm{~cm}$, the body length of a rat, see [3] for further details). Percentage time spent in three other equivalent locations (NW, SE, SW) was also calculated. In addition, the swimming pattern of the animals within their cue-based platform quadrants was observed, where percentage time of the initial $30 \mathrm{~s}$ spent in specific pre-defined areas was calculated. This included a corridor area (approximately $20 \mathrm{~cm}$ in width, inside the pool wall) and a platform corridor (a cor- ridor centred at the distance of the platform from the side, again approximately $20 \mathrm{~cm}$ in width, see Fig. 1a). A series of ANOVAs and post-hoc tests were performed to compare the percentage time spent in each of these pre-defined areas during retention.

All animals acquired the water maze, with mean escape latencies decreasing from $43.7 \pm 2.4 \mathrm{~s}$ on day 1 to $17.5 \pm$ $2.2 \mathrm{~s}$ on day 4 (Fig. 1b). A one-way between-groups ANOVA and post-hoc (Tukey) test confirmed this decrease to be significant $(F=20.31$, d.f. $=3, p<0.001)$. During the retention phase, one-way ANOVA and post-hoc (Tukey) tests revealed that animals in CT spent significantly more time searching for the platform in the NE quadrant compared to the SW or SE quadrants ( $39.16 \pm 4.67 \%, F=22.04$, d.f. $=3, p<0.001)$. On the contrary, CR followed the rotated cues to the SW quadrant, where they focused most of their search, again, compared to the NE or SE quadrant $(31.02 \pm 3.44 \%, F=10.62$, d.f. $=3, p<0.001$; Fig. 1c). Both groups spent a large percentage of time in the NW quadrant in which they were placed, particularly in the NW corridor area (Fig. 1c). No significant difference, however, was found between the two groups in the percentage time spent in this area $(t=0.724$, d.f. $=28, p>$ $0.05)$. Having determined from this that animals were using the distal visual cues in their search for the platform, we assessed the searching patterns of each group within their own cue-based platform quadrant (i.e. CT in NE quadrant and CR in SW quadrant; Fig. 1d). One-way ANOVAs indicated that there was a preference shown by $\mathrm{CR}$ for a particular location within their platform quadrant $(F=13.17$, d.f. $=3, p<0.001)$, which was not evident in CT $(F=1.179$, d.f. $=3, p>0.05$; Fig. 1d). Post-hoc (Tukey) tests revealed that CR spent most of their time swimming at the side of the pool in the corridor area $(p<0.01)$, as opposed to CT who did not display this behaviour.

The swimming patterns shown by the rats in the cuerotated condition indicate a tendency to swim at the side of the pool in the rotated quadrant, rather than searching in the accurate platform area. This tendency to swim around the side of the pool is termed thigmotaxis [2]. Although research has shown that olfactory and auditory cues may play a role in spatial navigation $[8,15]$, the literature indicates that these cues do not provide sufficient information by themselves for successful navigation, but must be presented in conjunction with a related visual cue $[14,15]$. We agree with this assertion and rule out a major contribution of these other sensory cues. However, we suggest that the observed thigmotaxic behaviour may be a result of internal vestibular conflict with the external visual sources.

The use of distal visual cues has been well documented as the main strategy used by navigating rats [12]. However, in the absence of useful visual cues, rats can orient in their environment using idiothetic navigation [18]. This idiothetic navigation or egocentric navigation is thought to rely on vestibular information [17]. Indeed Semenov and Bures [16] suggest that vestibular cues are important for orientation of the animal in the gravitation field and for estimation of an- 


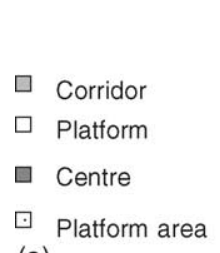

(a)

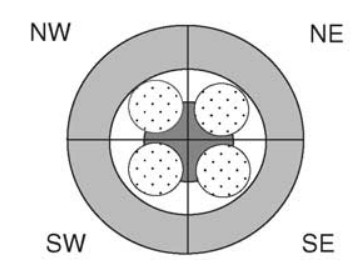

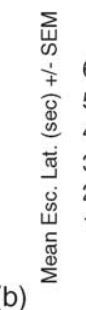

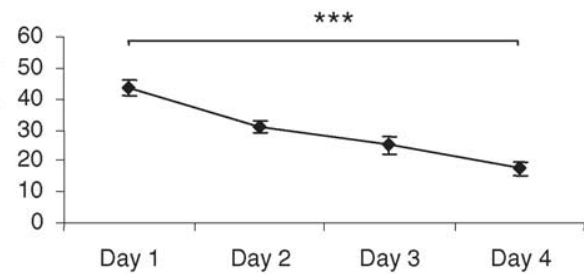

(c)

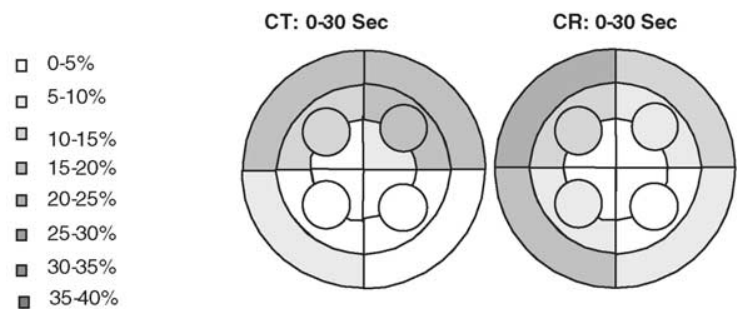

Mean \% time spent by CT in pre-defined areas of NE quadrant ( $0-30 \mathrm{sec})$

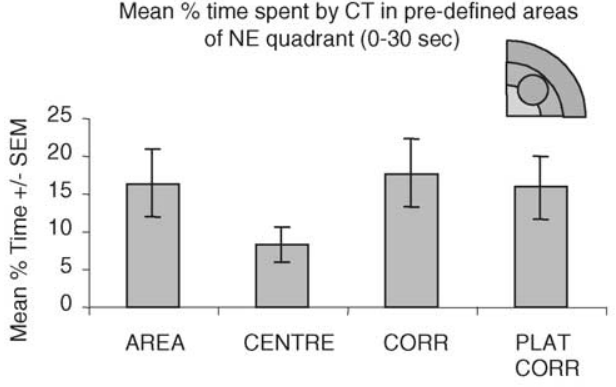

Mean \% time spent by $\mathrm{CR}$ in pre-defined areas of SW quadrant (0-30 sec)

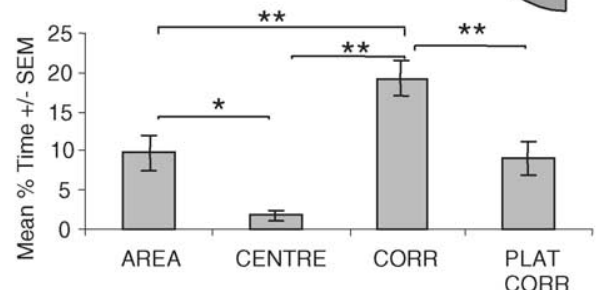

Fig. 1. (a) Pre-defined areas of measurement during retention probe trial. (b) Mean escape latency for acquisition phase. (c) Maze maps illustrating patterns of search for initial $30 \mathrm{~s}$ of retention phase. (d) Mean percentage time spent by animals in each pre-defined area of their platform quadrant (a) in the initial $30 \mathrm{~s}$ of retention.

gles between vectors plotted from the animal toward external landmarks. Both vestibular and visual information are, therefore, critical for efficient navigation [16]. We extend this by suggesting that both types of information are also critical for the retention of a spatial location and navigation towards this retained location. We further suggest that conflicting vestibular information to that given out by distal visual cues alone may have influenced CR's retention in the above experiment. While the vestibular information available to CT would direct them to the same quadrant as the allocentric, cue-based information, this would not be the case for CR. Instead, allocentric information available to $\mathrm{CR}$ would send them in the direction of SW quadrant, while vestibular information would direct them from their starting position in NW towards the NE quadrant. This conflicting information may have influenced CR's ability to accurately retain the water maze, where rats' ability to accurately locate the platform area was impaired. One method used to minimise vestibular information is via whole body rotations immediately before the retention trial (see [16]). We will in a second set of experiments attempt to examine the role played by vestibular information on spatial retention using this whole body rotation method.
Experiment 2: Animals received the same training procedure as those in experiment 1 (four trials per day for 4 days). Animals were placed into the maze from a pseudo-random starting point (N, S, E, W). Retention was assessed 7 days post-acquisition, where animals were randomly assigned to one of two groups: vestibular-rotated controls $(n=7)$ or the vestibular-rotated cue-rotated group $(n=7)$. Prior to being placed into the water maze, both groups were subjected to vestibular-rotation (see [4] for further details). This involved placing each individual animal into a small, square, plastic container $(18 \mathrm{~cm} \times 18 \mathrm{~cm} \times 18 \mathrm{~cm})$, which was then covered over with a dark cloth and rotated continuously, while at the same time being moved in a circuitous fashion around the room for approximately $20 \mathrm{~s}$. After rotation, the animal was immediately placed into the maze at $\mathrm{NW}$ and retention was assessed by removing the platform as before. For the vestibular-rotated control group (CT $+\mathrm{VR})$, the distal-cue layout remained the same as during the acquisition phase. For the vestibular-rotated cue-rotated group $(\mathrm{CR}+\mathrm{VR})$, the distal cues were rotated by $180^{\circ}$. Measures of retention again included an analysis of the percentage time spent in the platform quadrant (NE) and the cue-rotated, equivalent quadrant (SW) in the initial $30 \mathrm{~s}$. The swimming pattern of the animals 


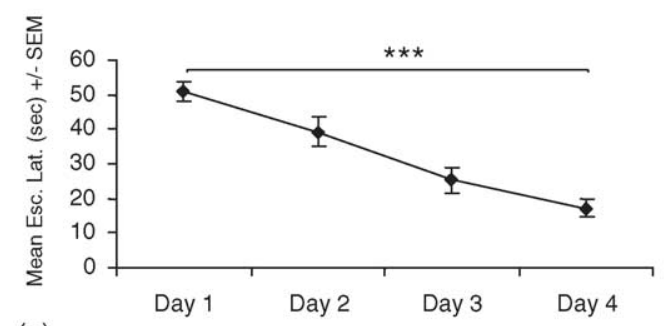

(a)

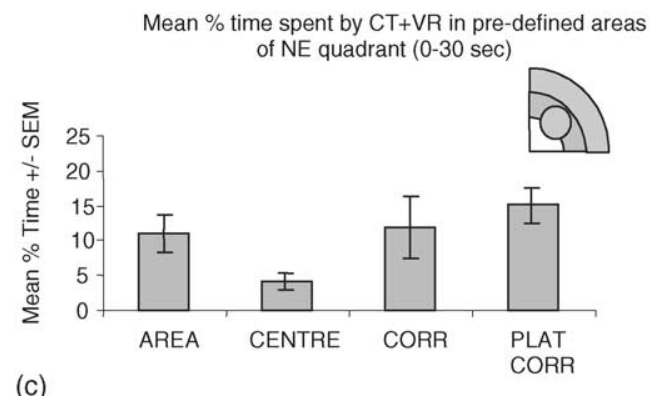

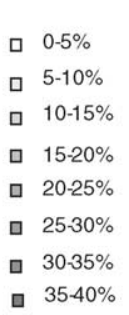

CT \&VR: 0-30 Sec

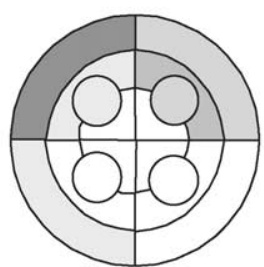

CR+VR: 0-30 Sec

(b)

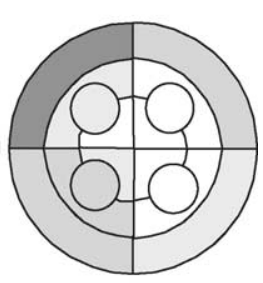

Mean \% time spent by CR+VR in pre-defined areas of SW quadrant $(0-30 \mathrm{sec})$

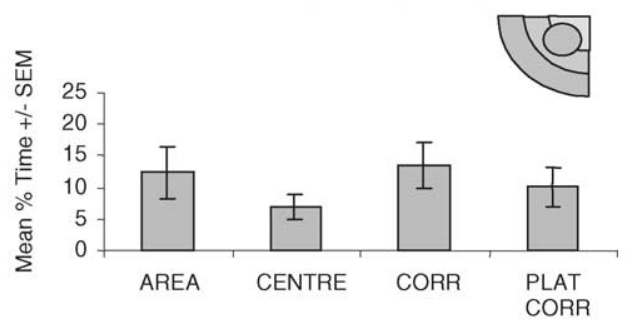

Fig. 2. (a) Mean escape latency for acquisition phase. (b) Maze maps illustrating patterns of search for initial $30 \mathrm{~s}$ of retention phase. (c) Mean percentage time spent by animals in each pre-defined area of their platform quadrant (see Fig. 1a) in the initial $30 \mathrm{~s}$ of retention.

within their cue-related platform quadrants themselves was observed, where percentage time spent in specific pre-defined areas was again calculated (see Fig. 1a). A series of ANOVAs and post-hoc tests were performed to compare the percentage time spent in each of these pre-defined areas during retention.

All animals acquired the water maze. One way betweengroups ANOVA and post-hoc (Tukey) tests revealed that mean escape latencies decreased significantly from $51.0 \pm$ $2.8 \mathrm{~s}$ on day 1 to $17.1 \pm 2.5 \mathrm{~s}$ on day $4(F=20.20$, d.f. $=$ $3, p<0.001$; Fig. 2a). During the retention phase, one-way between-groups ANOVA and post-hoc (Tukey) tests revealed that animals in $\mathrm{CT}+\mathrm{VR}$ spent significantly more time searching for the platform in the NE quadrant $(31.43 \pm 4.19 \%, F=$ 19.44, d.f. $=3, p<0.001)$ while $\mathrm{CR}+\mathrm{VR}$ followed the rotated cues, focusing most of their search in SW $(26.48 \pm 4.44 \%, F$ $=11.698$, d.f. $=3, p<0.001)$, in comparison to SE \& SW and NE \& SW quadrants, respectively (Fig. 2b). Animals spent a large amount of time where they were initially placed into the pool (NW corridor). This may be as a result of the animals' need to recover from the rotations before proceeding to locate the platform. No significant difference was noted between the groups for NW corridor $(t=0.279$, d.f. $=12, p>0.05)$. As in experiment 1 , the searching patterns of each group within their own cue-based platform quadrant was assessed. It was found that vestibular rotation (VR) removed the difference between searching patterns observed in CT $+\mathrm{VR}$ and those seen in CR+VR. Fig. 2c illustrates that, when $C T+V R$ and $\mathrm{CR}+\mathrm{VR}$ are compared, they show similar patterns of search. One-way ANOVAs revealed that neither CT + VR or CR + VR showed any preference for a particular location within their platform quadrant $(F=2.46$, d.f. $=3, p>0.05 ; F=0.76$, d.f. $=3, p>0.05$, respectively; Fig. $2 \mathrm{c}$ )
The results of this experiment highlight the influence of both external and internal information in spatial retention. While allocentric information is necessary for successful navigation and subsequent retention, our findings have shown that egocentric, vestibular information also plays an active role in these processes. Manipulations addressing vestibular influence in spatial navigation can have a marked effect on the long-term retention of a spatial environment. Animals following rotated visual cues must deal with conflicting information coming from vestibular sources. When the influence of this vestibular information is controlled through vestibular rotation, the difference between cue-rotated and control groups is removed.

These results support findings from the literature that, while spatial navigation is largely influenced by information received from external cues, internal vestibular information can also have an effect $[11,19]$. When egocentric information conflicts with that from allocentric cues, retention can be impaired, resulting in a less accurate searching strategy. However, manipulating the information that is being passed on from conflicting vestibular sources removes this effect, thus making navigation more accurate.

\section{Acknowledgements}

This work was supported by the Health Research Board of Ireland, the Department of Psychology, NUI Maynooth and the Irish Research Council for the Humanities and Social Sciences. We would also like to thank Mr. Alan Rogers of the Department of Electronic Engineering, NUI Maynooth, for his help in the initial stages of this research. 


\section{References}

[1] Aggleton JP, Vann SD, Oswald CJP, Good M. Identifying cortical inputs to the rat hippocampus that subserves allocentric spatial processes: a simple problem with a complex answer. Hippocampus 2000;10:466-74

[2] Barnett SA. The rat: a study in behaviour. Chicago: Aldine; 1963.

[3] Commins S, Cunningham L, Harvey D, Walsh D. Massed but not spaced training impairs spatial memory. Behav Brain Res 2003;139:215-23.

[4] Commins S, Gemmell C, Anderson M, Gigg J, O'Mara SM. Disorientation combined with bilateral parietal cortex lesions causes path integration deficits in the water maze. Behav Brain Res 1999;104:197-200.

[5] D'Hooge R, De Deyn PP. Applications of the Morris water maze in the study of learning and memory. Brain Res Rev 2001;36:60-90.

[6] Etienne AS, Sitbon S, Dahn-Hurni C, Maurer R. Golden hamsters on the eight-arm maze in light and darkness: the role of dead-reckoning. Q J Exp Psychol 1994;47:401-25.

[7] Gallistel CR. The organization of learning. Cambridge: MIT Press; 1990.

[8] Hill AJ, Best PJ. Effects of deafness and blindness on the spatial correlates of hippocampal unit activity in the rat. Exp Neurol 1981;74:204-17.

[9] McGauran AT, Harvey D, Cunningham L, Craig S, Commins S. Retention of cue-based associations in the water maze is time-dependent and sensitive to disruption by rotating the starting position. Behav Brain Res 2004;151:255-66.
[10] Moghaddam M, Bures J. Contribution of egocentric spatial memory to place navigation of rats in the Morris water maze. Behav Brain Res 1996;78:121-9.

[11] Morris RGM. Spatial localisation does not require the presence of local cues. Learn Motiv 1981;12:239-60.

[12] O'Keefe J, Nadel L. The hippocampus as a cognitive map. Oxford: Claredon Press; 1978.

[13] Pearce JM. Animal learning and cognition: an introduction. UK: Psychology Press; 1997.

[14] Rossier J, Haeberli C, Schenk F. Auditory cues support place navigation in rats when associated with a visual cue. Behav Brain Res 2000;117:209-14.

[15] Rossier J, Schenk F. Olfactory and/or visual cues for spatial navigation through ontogeny: olfactory cues enable the use of visual cues. Behav Neurosci 2003;117:412-25.

[16] Semenov LV, Bures J. Vestibular stimulation disrupts acquisition of place navigation in the Morris water tank task. Behav Neural Biol 1989;51:346-63.

[17] Stackman RW, Herbert AM. Rats with lesions of the vestibular system require a visual landmark for spatial navigation. Behav Brain Res 2002;128:27-40.

[18] Stuchlik A, Bures J. relative contribution of allothetic and idiothetic navigation to place avoidance on stable and rotating arenas in darkness. Behav Brain Res 2002;128:179-88.

[19] Vanderwolf $\mathrm{CH}$. The hippocampus as an olfacto-motor mechanism: were the classical anatomists right after all? Behav Brain Res 2001;127:25-47. 